\title{
¿Qué es un trauma abdominal y sus estudios?
}

Hernán Pérez Montesinos Luis Pérez Montesinos 

¿Qué es un trauma abdominal y sus estudios? 
Hernán Pérez Montesinos

Luis Pérez Montesinos 
¿Qué es un trauma abdominal y sus estudios?

(C) Hernán Pérez Montesinos Luis Pérez Montesinos

2021,

Publicado por acuerdo con los autores.

(C) 2021, Editorial Grupo Compás

Guayaquil-Ecuador

Grupo Compás apoya la protección del copyright, cada uno de sus textos han sido sometido a un proceso de evaluación por pares externos con base en la normativa del editorial.

El copyright estimula la creatividad, defiende la diversidad en el ámbito de las ideas y el conocimiento, promueve la libre expresión y favorece una cultura viva. Quedan rigurosamente prohibidas, bajo las sanciones en las leyes, la producción o almacenamiento total o parcial de la presente publicación, incluyendo el diseño de la portada, así como la transmisión de la misma por cualquiera de sus medios, tanto si es electrónico, como químico, mecánico, óptico, de grabación o bien de fotocopia, sin la autorización de los titulares del copyright.

Editado en Guayaquil - Ecuador

ISBN: 978-9942-33-373-5

DOI. https://doi.org/10.37959/se.voio.168

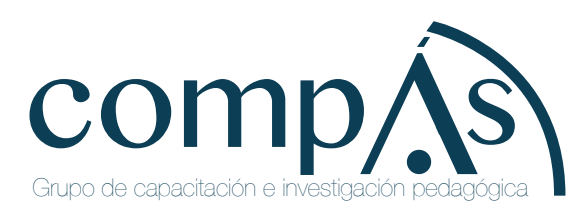


Cita.

Pérez, H., Pérez, L., (2021). ¿Qué es un trauma abdominal y sus estudios?. Editorial Grupo Compás. 


\section{Índice}

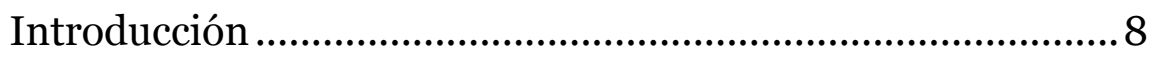

Capítulo I:..........................................................................

Mecanismos de lesión, trauma contuso o cerrado ...................11

Trauma penetrante................................................................... 13

Evaluación inicial ................................................................ 14

Capítulo II: Técnicas y métodos.............................................. 23

Tomografía Computadas (TC) ............................................24

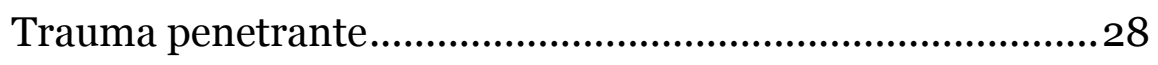

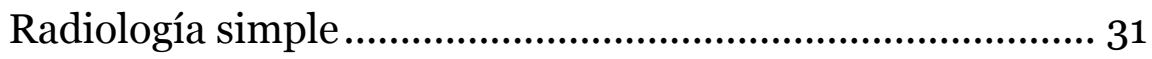

Lavado peritoneal diagnóstico (LPD) ..................................... 32

Laparoscopia diagnóstica....................................................... 34

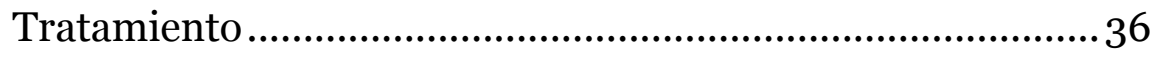

Capítulo III: Mecanismos y tipos ............................................... 38

FISIOPATOGENIA DEL TA.................................................. 41

Contractura de la pared abdominal ........................................46

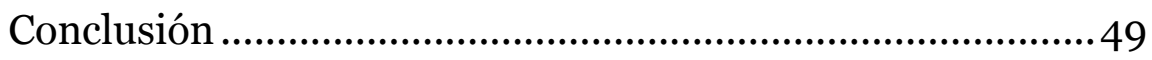




\section{Introducción}

Actualmente, el cuadro de traumatismo es la principal causa de muerte en los primeros cuarenta años de vida. En Ecuador, en 2000, entre las más de 30.000 muertes entre las edades de 15 y 64 años, casi 8.00o murieron por trauma. El mecanismo más común para recordar son los accidentes de tráfico (alrededor del 50\% de los casos), seguidos de las alturas, las heridas de bala, las puñaladas y las lesiones por aplastamiento (1) La mortalidad por lesiones masculinas es significativamente mayor. Esto se puede ver en todos los países de las Américas: Colombia tiene una relación hombre-mujer de 5,9, El Salvador 5,0, Chile 4,2, Estados Unidos y Canadá 2,9

y Cuba 2,4. Los accidentes de tráfico son el incidente violento más mortífero en Chile, matando a más de 2.000 personas cada año.

La epidemiología ha determinado que los accidentes no son accidentales y existen muchas condiciones que aumentan la 
probabilidad de accidentes. Estos factores provienen del entorno cultural humano, natural y social.

Reciente información epidemiológica procedente de Europa, Australia y Norte América, indica que los efectos combinados de control de velocidad, el uso obligatorio de cinturones de seguridad, bolsas de aire y tecnología avanzada en el frenado de vehículos, han contribuido a una redistribución del tipo de lesiones observadas en trauma abdominal; con menor incidencia de lesiones cerebrales severas, pero con un incremento de lesiones torácicas y abdominales. (2)Pese a que en los Estados Unidos la incidencia de lesiones abdominales penetrantes ha disminuido, no ocurre así en Chile, ni el resto de Latinoamérica (3)En Ecuador, los homicidios determinan un $11 \%$ de las muertes violentas, registrándose un claro incremento a partir de 1995. Nuestro país tiene una tasa de suicidios que supera el promedio latinoamericano, en el que predominan las lesiones autoinflingidas de los varones. 
En los servicios de emergencia, el traumatismo abdominal representa la mayoría de las operaciones quirúrgicas. En nuestro hospital, el Hospital Urgencia Asistencia Pública, representó el 10\% del total de traumatismos, de los cuales el $77,9 \%$ fueron traumatismos penetrantes, de los cuales el $88,3 \%$ fueron cuchillos y el 11,7\% armas de fuego (4). Cuando se habla de trauma abdominal, debe incluir diferentes formas de manifestaciones clínicas y complicaciones relacionadas. Incluye recursos que se pueden utilizar para hacer frente al diagnóstico de complicaciones. 
Capítulo I:

Mecanismos de lesión, trauma contuso o cerrado 
Es el resultado de la acción combinada de compresión, deformación, tensión y cortante. La magnitud de estas fuerzas está directamente relacionada con la masa de los objetos involucrados, su aceleración y desaceleración, y su dirección relativa durante el impacto. El daño ocurre cuando la suma de estas fuerzas excede la cohesión de los tejidos y órganos involucrados. Luego vino la constelación de hematomas, abrasiones, tejidos y órganos rotos y rotos. El choque directo y la presión son probablemente las causas más comunes de traumatismo mayor.

Si se conocen la fuerza y la dirección del impacto y el tamaño del área de contacto del paciente, se puede estimar la gravedad. También debemos considerar el trauma causado por la explosión. Aunque genera calor y quema y es penetrado por misiles auxiliares, su función principal es absorber la onda de explosión a través del cuerpo humano como una onda de choque. Esto puede causar picos de presión y ondas de choque, lo que ocasiona la mayor parte del daño. Los órganos más 
vulnerables son los que contienen gas, como los pulmones y los intestinos. En los traumatismos cerrados generales, los órganos con lesiones más graves son el hígado, el bazo, el mesentérico y el riñón. Si el paciente está atrapado en un vehículo con una "huella del cinturón de seguridad", debe sospecharse de una lesión por moco visceral.

\section{Trauma penetrante}

Las heridas punzantes y de bala a baja velocidad pueden dañar los tejidos a través de cortes o cortes. Las heridas de proyectil de alta velocidad transfieren mayor energía cinética a los órganos internos de la cavidad abdominal, tienen el efecto adicional de cavitación temporal y también causan lesiones abdominales, que se desvían y se rompen. Como heridas de arma blanca, nos referimos a objetos cortantes, como cuchillos de diferentes tamaños, cimitarras, cuellos, piolets o puñetazos.

Para un proyectil disparado por un arma de fuego, su daño depende no solo de la energía cinética (masa x velocidad), sino también de la estabilidad del proyectil (determinada por la 
ranura del cañón). Es importante distinguir entre heridas penetrantes producidas por armas de fuego $\mathrm{y}$ heridas penetrantes producidas por objetos cortantes. Estas heridas suelen tener trayectorias impredecibles debido a que las trayectorias son más directas y la relación anatómica entre el área penetrante y el daño del órgano interno es más cercana. En comparación con las heridas de bala, las heridas de bala requieren cirugía y es más probable que se vuelvan complicadas y mueran, mientras que las heridas con una sola mano tienen más probabilidades de requerir cirugía. Los órganos más vulnerables son el intestino delgado, el hígado, el estómago, el colon y las estructuras vasculares.

\section{Evaluación inicial}

Antes de darse cuenta de si existe una lesión intraabdominal, ya sea debido a un traumatismo cerrado o penetrante, el objetivo de los pacientes traumatizados debe ser detectar y tratar rápidamente la afección que amenaza la vida inmediata. 
En respuesta a la situación anterior, se debe implementar la primera revisión propuesta por ATLS, la reanimación y restauración de funciones importantes, la segunda revisión y los principios de tratamiento de la lesión definitiva. (Soporte vital avanzado para traumatismos) American College of Surgeons.

A continuación, en pacientes con hipotensión, el objetivo es determinar rápidamente si existe una lesión abdominal y si esta es la causa de la hipotensión. Para los pacientes con hemodinámica normal sin signos de peritonitis, se puede realizar una evaluación más detallada para determinar si hay lesiones específicas durante el período de observación o signos de peritonitis o sangrado.

Examen físico: la historia clínica y el examen físico pueden indicar una posible lesión intraabdominal. La información obtenida del personal prehospitalario involucrado en la escena del trauma es importante. El tipo de fuerza aplicada, el área probable de impacto, la altura de la caída, la deformación del 
vehículo, el uso de cinturones de seguridad u otros dispositivos de seguridad, pueden levantar la sospecha de una posible lesión intraabdominal. La información sobre signos vitales, lesiones aparentes y respuesta al tratamiento prehospitalario también se puede obtener de la persona que realiza el manejo prehospitalario.

Después de que un paciente sufre una lesión contusa, un examen físico del abdomen generalmente no es confiable porque casi la mitad de los pacientes sin signos de examen físico tienen una laparoscopia positiva. Los cambios en el nivel de conciencia, las lesiones de la médula espinal u otras lesiones que distraen, además de los efectos de ciertas drogas u otras sustancias como el alcohol, pueden dificultar aún más el examen clínico. Incluso en muchos centros, la exploración física no es la primera en el abordaje multidisciplinario y la ecografía sí lo es. Durante el examen: El paciente debe estar completamente desnudo y el examen debe incluir toda la espalda, las axilas y el perineo. 
No se debe omitir registrar el pulso circundante, el tacto rectal y el examen genital. Visualice todas las "marcas" que sugieran una lesión, equimosis abdominal o perineal, que pueden indicar una fractura pélvica. Distensión abdominal e irritación peritoneal. En las heridas penetrantes se pueden encontrar evidencias de vísceras intestinales o epiplón. Investigar el estado del embarazo. No olvide colocar la sonda gástrica y el catéter urinario, que nos pueden brindar información relevante. El tubo de estómago evita la hinchazón y reduce el riesgo de aspiración bronquial. Los catéteres uretrales son útiles para la reanimación, pero la colocación transuretral está prohibida para pacientes con sospecha de daño uretral. Se trata de pacientes con fracturas pélvicas, con sangre en la uretra, equimosis perineal, sangre en el escroto o próstata agrandada o indistinta durante el tacto rectal.

Investigación y gestión: se habla a modo de enseñanza, independiente del estudio de cada mecanismo, porque si bien 
los métodos de diagnóstico son los mismos, tienen usos diferentes.

Lesión cerrada: La primera es identificar a aquellos pacientes que tienen indicaciones inmediatas de laparotomía.

Los pacientes con inestabilidad: hemodinámica han recibido ultrasonido o lavado peritoneal diagnóstico a pesar de una reanimación inicial adecuada.

Aunque la exploración física de estos pacientes puede aportar una gran contribución, la sensibilidad en la detección de lesiones intraabdominales es de alrededor del $65 \%$. Los exámenes físicos repetidos aumentarán este valor. Diferentes autores coinciden en la confiabilidad del examen físico inicial. Las pruebas de laboratorio tienen poco valor. El bajo hematocrito asociado con la inestabilidad hemodinámica indica la presencia de hemorragia, pero no se especifica la ubicación. Un valor dentro del rango normal no excluye daños. De manera similar, el nivel de amilasa o lipasa tiene poca sensibilidad y especificidad. Pueden ser normales o estar 
alterados y no indican ni excluyen una lesión pancreática (3, 10). Un gas arterial y una base insuficientes pueden indicar acidosis debida al choque e indicar la necesidad de reanimación. El analizador de aliento debe considerarse desde la perspectiva del derecho médico.

Según el mecanismo de lesión, las pruebas de diagnóstico de la lesión relacionada y la estabilidad hemodinámica. Estudios radiológicos simples: forman parte del plan de manejo y pueden aportar información directa, como rotura de músculo superior o neumoperitoneo, o indirectamente, información como fracturas de columna lumbar o costillas inferiores, que pueden orientarnos hacia la lesión. 

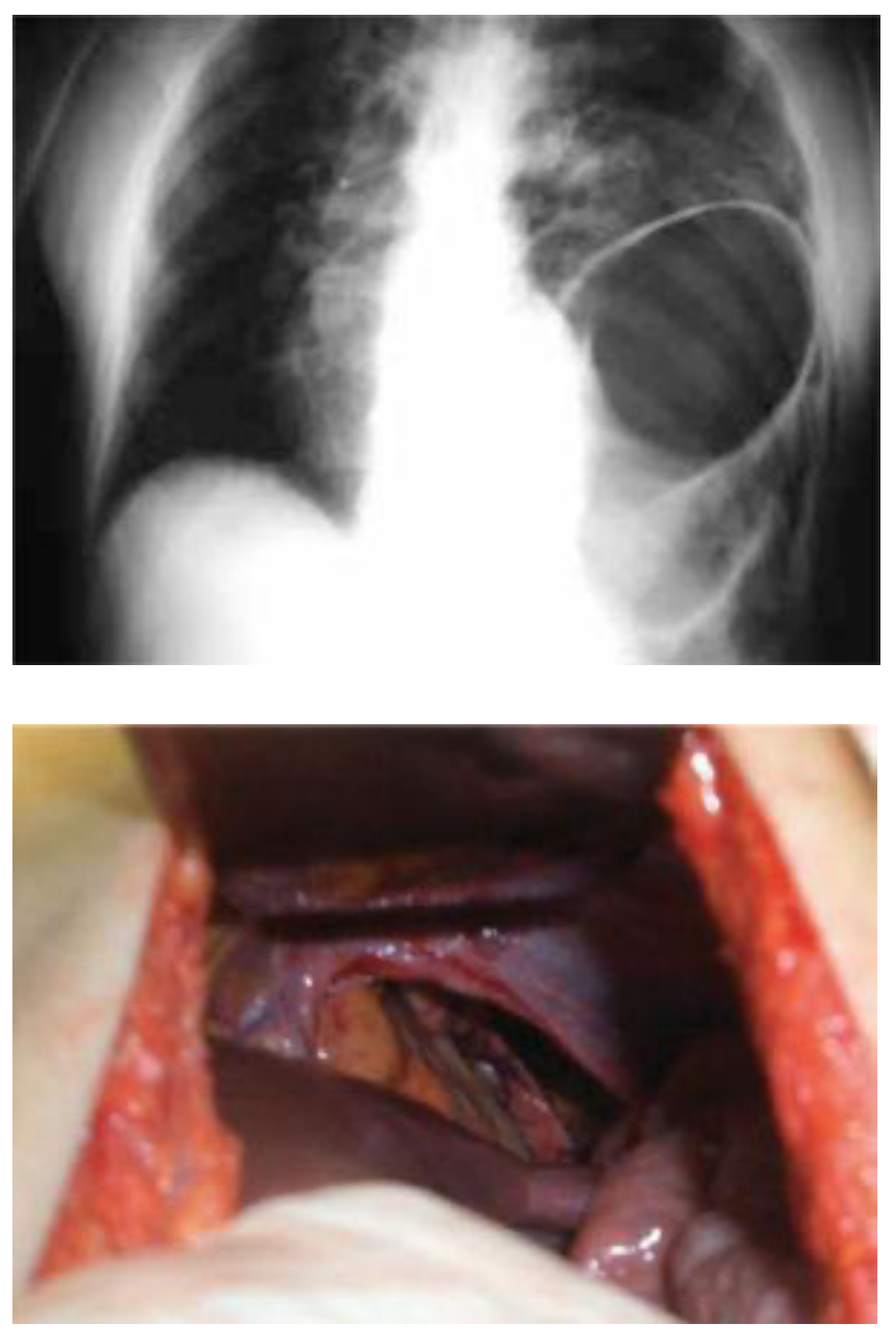
Ultrasonido: FAST: Concéntrese en la ecografía abdominal para verificar si hay traumatismo. Este es un procedimiento rápido y no invasivo que es fácil de realizar y explicar en el área de emergencia por cirujanos capacitados. Su finalidad es determinar la presencia de sangrado peritoneal, por lo que ha sustituido a la LPD en muchos centros asistenciales. Su sensibilidad y especificidad varían de un centro a otro, siempre cerca del 95\%, llegando incluso al 100\% en las publicaciones nacionales. En pacientes obesos con enfisema subcutáneo y en pacientes con cirugía previa, su efectividad está comprometida, y no es confiable en la perforación intestinal. La contraindicación de este método es la evidente necesidad de una laparotomía o la falta de personal adecuado para la laparotomía. El principio se basa en la detección de líquido en cuatro áreas específicas del abdomen: periliver, pericardio, perispleen y pelvis. 
Lavado peritoneal diagnóstico: Posteriormente reemplazó muchas punciones abdominales falsas negativas y positivas. El diagnóstico de lesiones intraabdominales en pacientes con traumatismo cerrado es un método rápido y preciso, pero con la llegada de la ecografía, la frecuencia de las ecografías es cada vez menor. Su uso temprano ayuda a realizar una laparotomía más rápida y rápidamente, reducir la pérdida de sangre y evitar la transfusión de sangre. Su desventaja es que es un método invasivo con baja especificidad. En nuestro centro, nunca ganó seguidores. Sigue siendo útil cuando el paciente no responde adecuadamente a la reanimación y el examen ecográfico es negativo. No se pueden detectar el músculo diafragma y las lesiones retroperitoneales. 
Capítulo II: Técnicas y métodos 
Técnica: Inserte un catéter en la cavidad peritoneal para succionar sangre o líquido. Esto se puede hacer mediante tecnología abierta, semiabierta o cerrada. Si no se quita nada, inyecte 1 solución salina normal tibia (o $10 \mathrm{ml} / \mathrm{kg}$ para niños). Asegúrese de mezclar el líquido, comprimir el abdomen y rotar al paciente, vaciar el líquido por gravedad y enviar la muestra al laboratorio para su análisis. Se considera positivo:

-Extracción de $10 \mathrm{ml}$ de sangre roja al aspirar

•>100.00o glóbulos rojos /cc

•>500 glóbulos blancos /cc

-Presencia de bacterias

-Presencia de bilis

-Presencia de partículas de comida

\section{Tomografía Computadas (TC)}

Es una herramienta importante para el tratamiento de traumatismos pélvicos abdominales y abdominales cerrados. 
Reservados para pacientes con hemodinámica estable, estos pacientes pueden ser transferidos a tomografía y pueden tolerar el estudio. Deben usarse agentes de contraste orales e intravenosos. La TC puede determinar la presencia de órganos sólidos, derrame abdominal, sangre, lesiones aéreas y órganos retroperitoneales, estas lesiones pueden haber sufrido un traumatismo y no ocasionaron sangrado peritoneal, por lo que no pueden ser detectadas por ecografía también muestra el grado de lesiones de estructuras como el bazo y el hígado, y determina la extravasación del medio de contraste, lo que significa sangrado activo.

Por estas características, la TC es muy útil para determinar el tratamiento y manejo de las lesiones. En cualquier caso, el valor de los contrastes orales en el diagnóstico de perforación visceral hueca es cuestionable y está relacionado con el riesgo de inhalación bronquial, por lo que no debe realizarse de forma rutinaria. La sensibilidad de la TC para detectar lesiones de órganos sólidos es del 92-98\% y la especificidad se acerca al 
99\%. No puede detectar lesiones del músculo diafragma, intestino y cierto páncreas. Se debe considerar la experiencia del radiólogo al explicar el examen.

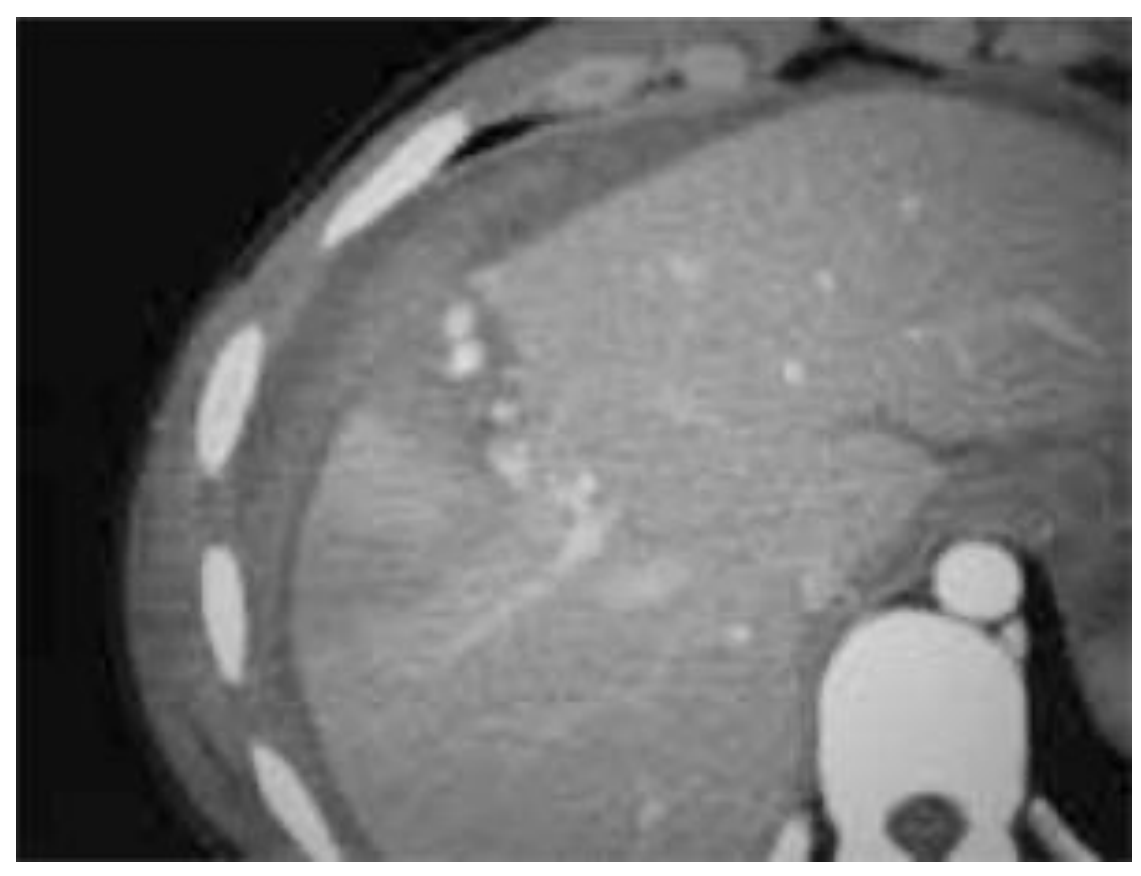

Figura 1: Trauma hepático. 


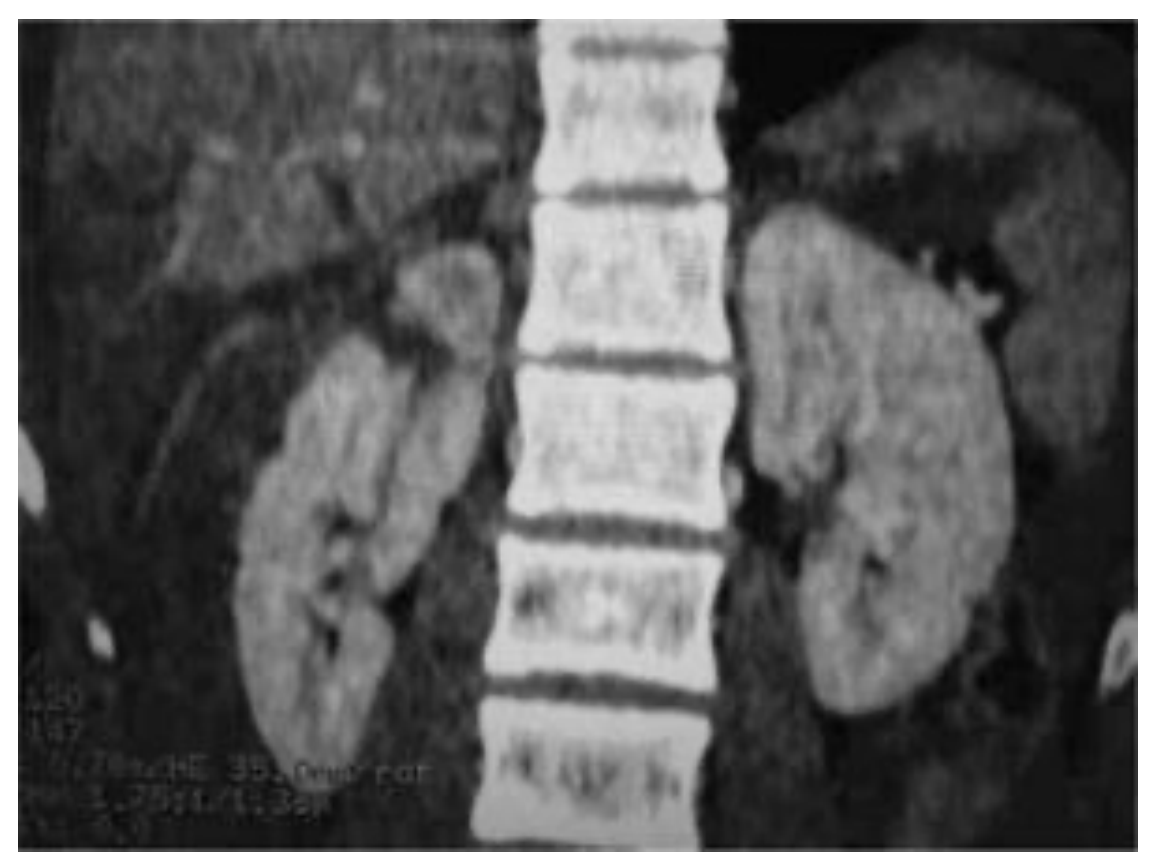

Figura 2: Trauma renal polo superior derecho.

Laparoscopia diagnóstica: debido a su alto costo, su aplicación en traumatismos abdominales cerrados es limitada. Sin embargo, es útil para reducir el número de cirugías abiertas no terapéuticas y evitar la cirugía abierta tardía. Es útil cuando el estudio preliminar del paciente muestra que la enfermedad no es lo suficientemente grave como para requerir una 
laparotomía de rutina (15). Según nuestra experiencia, también es útil cuando el paciente se encuentra en planta por traumatismo por otras causas, como fractura expuesta, hematoma epidural, etc., y presenta hipotensión desconocida. Otras aplicaciones son terapéuticas.

Los estudios radiológicos especiales son útiles cuando el paciente tiene hematuria. Cuando necesitamos extirpar el riñón y queremos conocer la función del riñón contralateral, también se puede utilizar en el pabellón. En comparación con el traumatismo abdominal y pélvico, la cistoscopia debe realizarse cuando se sospecha una fractura de uretra.

\section{Trauma penetrante}

Después de excluir pacientes con indicaciones claras de cirugía, como shock, órganos internos, signos de peritonitis o sangre en el estómago (detectado cuando se coloca una sonda gástrica), recto (detectado por un tacto rectal) o vejiga (detectado por un catéter). Puede estudiarse más a fondo. Como sugieren varios autores, los pacientes que incluyen 
heridas de bala pueden incluirse en las guías de auge como otra indicación. Las razones citadas son: más del 90\% de las lesiones graves, la laparotomía innecesaria es una operación inofensiva y el examen físico no es confiable.

Sin embargo, existen algunas excepciones en pacientes hemodinámicamente estables, como heridas de proyectil de baja velocidad, heridas tangenciales o laterales, según diferentes autores, que permiten otros métodos diagnósticos. Según Demetriades et al., Los órganos internos del epiplón mayor no son una indicación clara de laparotomía, este es nuestro estándar común, pero debemos recordarlo porque está relacionado con el daño del intestino delgado. En esta serie de estudios, en 24 pacientes con órganos internos omentales (limpiados, bandeados y devueltos a la cavidad), todos los pacientes fueron hospitalizados y solo 1 requirió laparotomía. Los pacientes deben considerar especialmente las armas "in situ", que deben sacarse de la sala de exposiciones con el abdomen abierto y visualizado directamente. 
Examen físico: el paciente debe ser examinado cuidadosamente para evitar cualquier lesión. Las balas que no atraviesan huesos u otros objetos sólidos suelen moverse en línea recta, pero debemos recordar que el camino es impredecible. Preste atención a las anomalías en el cuerpo, el perineo y el recto. Se debe calcular y evaluar el número de heridas de bala. Un número impar significa que hay una bala en el paciente. Se debe palpar el abdomen en busca de sensibilidad y se debe realizar un examen neurológico para descartar una lesión de la médula espinal.

En heridas penetrantes provocadas por pinchazos $\mathrm{u}$ otros objetos punzantes, siempre que se sospeche el poder de penetración, la herida puede explorarse siempre que se ubique por debajo del margen costal y delante de la línea axilar anterior. Para los pacientes que están a punto de morir, es técnicamente imposible explorar heridas (como heridas por arma de fuego de carga múltiple), heridas penetrantes obvias (como rasguños viscerales) y heridas por "inflación" no deben 
explorarse. Debido al grosor de los músculos de esta zona, no tiene efecto en las heridas de la parte posterior del abdomen. La exploración se realiza bajo anestesia local y se inserta un dedo enguantado. Si se confirma que ha penetrado la fascia del recto posterior del abdomen, se debe hospitalizar para continuar el estudio realizando un examen físico continuo (el valor es variable, pero si no, sigue siendo de gran utilidad) otros métodos de diagnóstico disponibles), o con los métodos que a continuación se sugieren:

\section{Radiología simple}

Ayuda a determinar la trayectoria de las balas, lo que se facilita marcando los orificios en la piel con marcadores radiopacos. También ayuda a ubicar el proyectil si no hay salida, lo que hace sospechar las lesiones causadas. Debemos recordar que el proyectil puede ingresar al cuerpo por un área distinta al abdomen y sin embargo lesionarlo. Se debe sospechar de los proyectiles que ingresan en cualquier área desde el cuello hasta 
la porción media de los muslos. Ayuda a detectar neumoperitoneo, fracturas de columna, neumo o hemotórax

\section{Lavado peritoneal diagnóstico (LPD)}

La sensibilidad, especificidad y certeza del examen penetrante abdominal fueron del 95,9\% y el 98\%, respectivamente. Sin embargo, muchos autores creen que la "sensibilidad excesiva" es el problema porque provoca una cirugía abierta innecesaria. Además, se sostiene que este es un proceso que requiere mucho tiempo, es costoso e innecesario, y también puede causar enfermedades iatrogénicas, porque estabilizar una pequeña cantidad de sangre en la cavidad abdominal del paciente no es necesariamente una indicación de cirugía. Por otro lado, se concluyó que en pacientes con heridas causadas por escopeta, no hay indicación para laparotomía inmediata, y LPD es un indicador confiable de lesión intraabdominal y laparotomía.

Ecografía: su uso como método diagnóstico para el traumatismo abdominal penetrante es controvertido. Si bien muchas personas creen que tiene poco efecto en pacientes 
estables, si el resultado es positivo se puede inferir que existe daño orgánico interno. También se considera útil para pacientes que están borrachos y cuyo examen físico no es confiable. Su uso es fundamental en heridas próximas al corazón o tórax y abdomen para diagnosticar de inmediato un posible sobredimensionamiento pericárdico o taponamiento cardíaco

TC

Debido a una mejor definición de las imágenes y una mayor velocidad de investigación, este método de diagnóstico es más común en la actualidad. Este estudio requiere la estabilidad hemodinámica del paciente. Existen publicaciones que revelan la utilidad de la invasión peritoneal y el daño visceral (incluida la invasión peritoneal y el daño visceral) en heridas laterales y posteriores, el examen utiliza tres comparaciones, inyecciones orales e intravenosas. El recto y las heridas de bala pueden proporcionar información valiosa para ayudar a los cirujanos experimentados a realizar un tratamiento selectivo de estos 
pacientes. Todavía se consideran rumores, pero deben considerarse.

\section{Laparoscopia diagnóstica}

Existe suficiente evidencia científica para respaldar su uso. Tiene varias ventajas: puede ver directamente el órgano lesionado y puede tratarse, y puede sospechar indirectamente otras complicaciones. En comparación con los pacientes que necesitan cirugía abierta, la estancia hospitalaria se reduce. El mayor beneficio es la reducción de la cirugía laparoscópica no terapéutica, que tiene una mayor morbilidad e incluso mortalidad en traumatismos. Detecta claramente la penetración peritoneal en heridas de bala tangenciales o lesiones laterales.

Es el método preferido para detectar las lesiones recesivas del músculo del tórax y las heridas del abdomen. Desventajas: El uso de anestesia general, alto costo económico, requiere un equipo quirúrgico capacitado en tecnología y trauma. La disponibilidad del instrumento, el riesgo de neumotórax, 
lesión accidental o el riesgo causado durante la cirugía. Vale la pena discutir todas estas deficiencias, y no todos los autores están de acuerdo con ellas.

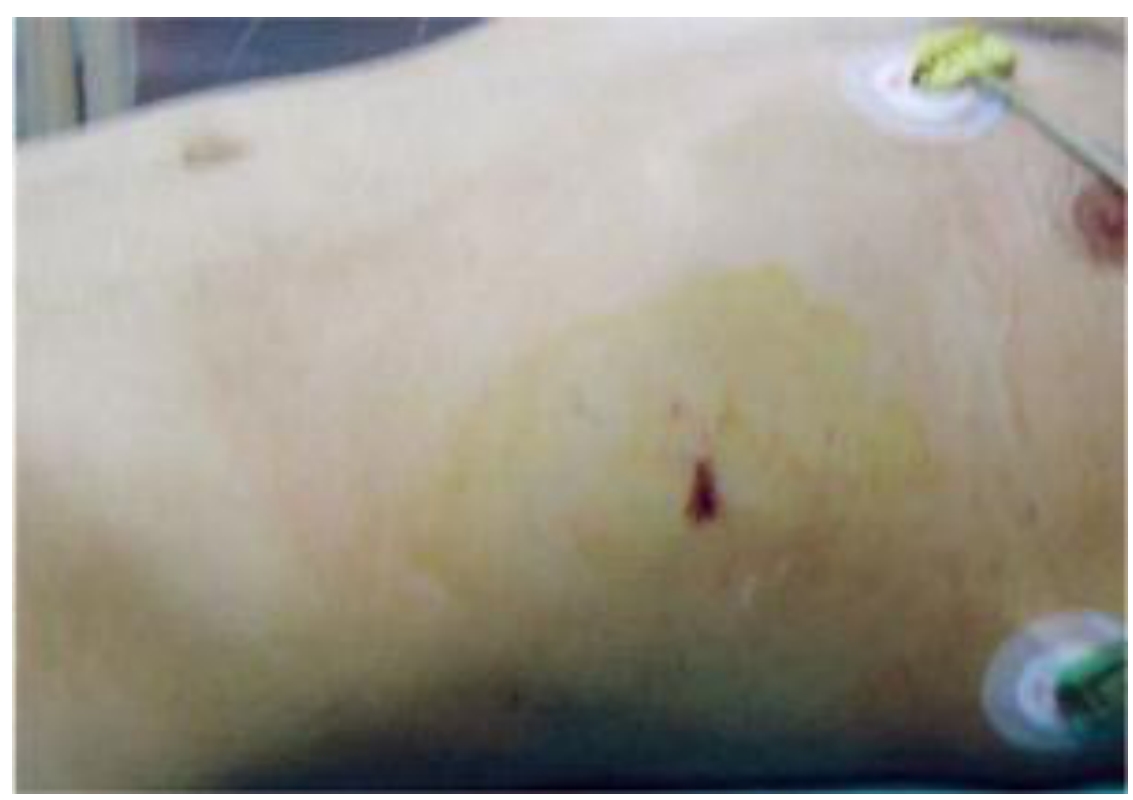

Figura 3: Herida tóraco-abdominal. 


\section{Tratamiento}

Como se mencionó anteriormente, los pacientes con traumatismo abdominal que son hemodinámicamente inestables o tienen signos evidentes de peritonitis deben ser reanimados de inmediato en la sala de emergencias y someterse a cirugía de inmediato. Según la gravedad y severidad del daño encontrado, se puede realizar una cirugía convencional, es decir, para identificar y tratar cada tipo de daño, o realizar una cirugía de control de daños. Esto último incluye controlar el sangrado y la contaminación, cerrar temporalmente el abdomen y luego resucitar en la unidad de cuidados intensivos para reparar la herida de la segunda forma.

De acuerdo con las regulaciones ministeriales de Ecuador, los pacientes deben recibir prevención del tétanos y profilaxis con antibióticos, y se debe establecer un calendario de lesiones adecuado. Generalmente indicado en traumatismos abdominales penetrantes y cirugía abdominal. Los pacientes 
con hemodinámica estable deben ser evaluados y se deben utilizar los métodos de diagnóstico descritos En base a los hallazgos, se debe realizar un tratamiento conservador o una cirugía para reparar órganos específicos.

En cuanto al tratamiento conservador propuesto para los órganos internos, se deben cumplir algunos requisitos básicos: estabilidad hemodinámica, ausencia de signos peritoneales y ausencia de transfusión sanguínea continua. El éxito depende del órgano lesionado, la diferencia en el hígado está entre el 50 y el 80\% y la diferencia en el bazo está entre el 65\%. Solo el 10\% de los riñones requieren reparación quirúrgica. Existe una variedad de algoritmos que pueden tomar decisiones en el manejo de estos pacientes, y estos algoritmos se pueden ajustar según la situación real de cada centro. También tenemos un acuerdo en el que todos los pacientes lesionados en la zona torácica abdominal izquierda serán explorados por vía laparoscópica. Cabe señalar que, en muchos centros, la laparotomía de rutina se sigue realizando a todos los pacientes 
con lesiones abdominales, especialmente lumbares, porque estos pacientes pueden tener lesiones de colon no intencionales

\section{Capítulo III: Mecanismos y tipos}




\section{Mecanismos}

Cuando agentes traumáticas golpean el abdomen, pueden ser directas, como patadas, golpes de timón, cuernos, armas de fuego, etc. El mecanismo indirecto es el retroceso, caída desde altura $\mathrm{o}$ alta presión en el abdomen.

\section{Tipos}

Puede ser de dos tipos:

a) Lesión contundente o contusión, llamada contusión. Se caracteriza por una solución que no presenta continuidad en la pared abdominal. El medio que produce esta sustancia es una superficie roma o plana, el tipo de timón, puño, etc.

b) Las heridas abiertas o penetrantes se denominan heridas. En este caso, se crea continuidad en la pared abdominal mediante el corte o fijación de elementos por arma de fuego o arma blanca. La herida solo puede afectar la pared abdominal y los órganos internos de la cavidad abdominal, con el peritoneo como límite y el peritoneo en sí es el límite de la 
cavidad abdominal. En base a este elemento, podemos tomar como ejemplo la clasificación de heridas de Quénu, que es muy práctica.

Las heridas no penetrantes son heridas que no desplazan el peritoneo, por lo que no llegan a la cavidad abdominal. Los penetrantes son los que destruyen las caries. Ante la TA, se debe considerar la pared o los órganos internos en el momento del trauma. Las paredes que se contraen pueden desempeñar un papel protector para prevenir lesiones graves; las paredes relajadas pueden ayudarlos. En órganos internos sólidos con exceso de sangre o bilis, se pueden promover daños más graves que en pacientes sin estasis. Un órgano cardíaco vacío en un estado lleno puede incluso romperse, mientras que un órgano vacío no sufrirá mucho daño. Asimismo, se debe considerar la enfermedad previa o el estado normal de los órganos internos. Los tumores sólidos, los parásitos o los órganos internos infectados y los órganos internos huecos gravemente enfermos son mucho más dañinos para el trauma. 


\section{FISIOPATOGENIA DEL TA}

En la TA, algunas partes de la pared abdominal se lesionarán gravemente hasta cierto punto, independientemente de si hay contenido abdominal involucrado. Es decir, órganos internos sólidos o huecos, medios y vasos sanguíneos, páncreas biliar o uretra. En la pared se pueden observar lesiones leves, como equimosis, eccema, hematoma, neuroma acelular roto, músculo, seguido de infección, seguido de celulitis, tumor de tallo o hematoma infectado, que puede conducir a absceso o esputo. Especialmente. Cuando sean heridas o hematomas con erosión cutánea. Estas imágenes generalmente requieren cirugía para evacuar, drenar, reparar, etc.

Hacia la cavidad, los órganos internos y los catéteres sufrirán diferentes tipos de lesiones. Los órganos internos sólidos son más vulnerables a las lesiones debido a la fragilidad y el volumen de su parénquima. Cada órgano interno se comporta de manera diferente cuando se lesiona.

\section{Hígado}


En el hígado, puede haber un hematoma superficial debajo del líquido seroso o un hematoma pequeño, mediano o grande que afecta un lóbulo. Los hematomas subserosos pueden convertirse en hematomas anatómicos progresivos, que pueden conducir a una inmersión sustancial. Los pequeños hematomas centrales se pueden organizar o absorber. Los hematomas medianos y grandes requieren un drenaje quirúrgico abierto bajo el control de una ecografía o un drenaje transparietal mediante punción en el dedal.

Existen roturas sustanciales o lesiones sólidas de tipo lagrimeo, que pueden ser de tipo regular, es decir, bordes afilados, o roturas irregulares o irregulares, curvas o estrelladas, daños en venas, arterias o vías biliares, en ocasiones acompañadas de separación sustancial; en otras ocasiones, el parénquima puede aplastarse en muy pequeña o gran extensión, dando una sensación blanda. Estas lesiones hacen que la sangre se filtre al espacio libre o que la bilis se filtre, cuya frecuencia es ambas. Dependiendo de la situación, 
se llama peritoneo, vesícula biliar o epitelio de la vesícula biliar.

El tratamiento de la AT con enfermedad hepática incluye cirugía, práctica de ligadura, sutura, reparación y extirpación de ganglios o lóbulos hepáticos; cuando estos procedimientos no son factibles, se pueden usar envases cerrados o abiertos, y campos quirúrgicos. El método cerrado requiere cirugía después de 8 o 10 días para eliminar los componentes usados. El campo abierto estilo Mikulitz debe retirarse del campo o la hoja mediante tracción gradual durante $4 \quad \begin{array}{llll}4 & 5 & \text { días }\end{array}$ consecutivos.

\section{Bazo}

Cuando el bazo está traumatizado, puede ocurrir una cantidad muy pequeña de hematoma subseroso, el parénquima esplénico y las cápsulas de diferentes tamaños se rompen y el bazo se destruye por completo, dejando solo los vasos pediculares. Cuando el trauma es severo, el flujo sanguíneo en la cavidad abdominal será intenso y aparecerán 
inmediatamente signos de hipovolemia y descompensación de signos vitales. Por otro lado, cuando el grado de las lesiones del bazo es pequeño, el hematoma periesplénico progresivo suele progresar hasta superar la capacidad de las células del bazo para mantener los signos vitales normales. Muchas personas no presentan signos de hipovolemia durante varias horas o incluso días. Cuando el hematoma se vacía de repente, la compensación se desequilibra.

Este método se llama "hemorragia en dos etapas" y se caracteriza solo por el bazo. Debido a la fragilidad del parénquima, el tratamiento quirúrgico conservador del bazo dañado mediante suturas, compresión del epiplón o gelatina no es satisfactorio; aunque existen consecuencias inmunológicas, se suele extirpar todo el órgano.

\section{Páncreas}

Los órganos que se encuentran en la columna vertebral (LI-L2) son vulnerables a traumatismos en la parte superior del abdomen. Puede causar hematomas retroperitoneales que son 
difíciles de diagnosticar y tratar. El traumatismo parenquimatoso del páncreas causado por traumatismo o contusión provocado por pancreatitis traumática se manifiesta como dolor intenso, vómitos y obstrucción intestinal adinámica, que puede superarse con medicación. Por lo general, generalmente se instala otro seudoquiste pancreático, que se tratará quirúrgicamente para drenar el estómago o el yeyuno internamente.

\section{Riñones}

Cuando el riñón está traumatizado, solo puede haber lesiones del parénquima renal y / o de la pelvis renal, la vía biliar y el sistema ureteral. Cuando la lesión es solo una lesión sustancial, el resultado es un hematoma perirrenal, a veces de gran amplitud, que puede originar la bóveda lumbar y ser provocado por un coágulo. Puede sorprenderse antes de eso. Atribuible a la afectación de las glándulas suprarrenales. Si la lesión es del tracto urinario, la orina también se extravasará a las células renales, lo que producirá una fuerte reacción en el 
tejido de las células grasas, inflamará el área y provocará un dolor intenso, lo que provocará una infección del hematoma del tracto urinario. El tratamiento es cirugía, que incluye limpieza, drenaje y reparación de la enfermedad. En general, esta actitud debería ser muy conservadora. Cuando el órgano no se pueda guardar y restaurar, retírelo.

\section{Contractura de la pared abdominal}

Según la edad y el sexo, la pared abdominal de todas las personas tiene un estado normal. Sin embargo, en pacientes que sufren un traumatismo, debido al reflejo visceral secundario a la lesión peritoneal, este estado cambiará a una contractura parcial o sistémica. Puede cambiar de semicontractura a contractura muy severa, tipo "abdomen en mesa", especialmente en niños o jóvenes. El anciano casi nunca respondió. Este reflejo se agotará en el transcurso de 48 a 72 horas, pudiendo entrar en la etapa de "atrofia abdominal", es decir, relajación completa por hipotonicidad, lo que puede dar lugar a errores de interpretación. Por lo tanto, la pared 
abdominal debe revisarse repetidamente para desarrollar tales signos en unas pocas horas.

La inactividad muscular: señal característica del traumatismo abdominal, evidenciada por una respiración muy superficial (preferiblemente de costillas), para evitar movilizar el abdomen para evitar el dolor. Se acompaña de pólipos. El abdomen lesionado puede tener signos típicos, como lesión pancreática o equimosis periférica (halo violeta alrededor del ombligo) cuando se rompe la parte posterior del duodeno. El signo de Job es producto de la inserción de aire en el hígado y el espacio renal, lo cual está clínicamente indicado por la desaparición de la insensibilidad hepática por H.D. , Se convierte en un golpeteo.

La impresión clínica debe confirmarse mediante rayos X. Un abdomen simple, postura de pie, muestra que hay un halo de presión entre el $\mathrm{pH}$ horizontal y la superficie del hígado. Cuando la perforación está en el estómago o el duodeno, ocurre antes, y cuando el aire sale del recto o del colon sigmoide y del 
colon superior, ocurre más tarde. La brusquedad de la pared abdominal se puede desplazar con la movilización del paciente, y existe líquido libre en la cavidad abdominal, tipo, sangre, orina, bilis, etc., ajustando así la contundencia. Síntomas generales: los pacientes traumatizados pueden experimentar cambios de temperatura, constantes de hemoglobina y constantes vitales. En la fase de choque inicial, puede ocurrir hipotermia, luego volver a la normalidad y luego puede aumentar a $38^{\circ}$ o $38,5^{\circ}$. En el caso de la infección peritoneal, se puede ver después de un promedio de 6 horas.

La constante de glóbulos cambia constantemente, cuando hay extravasación de sangre, el hematocrito disminuye y el número de glóbulos rojos disminuye y puede ocurrir un shock hipovolémico. Los signos vitales, el tipo de pulso, la presión arterial y la diuresis son datos que deben monitorearse continuamente en AT; le advierten de inmediato que instale imágenes de sangrado intraperitoneal. Cuando estos cambios 
son importantes, se deben tomar medidas antes de que pueda ocurrir una pérdida irreversible de compensación.

\section{Conclusión}

El tratamiento del traumatismo abdominal continúa suscitando interés y controversia. Hay muchas ayudas de diagnóstico y varios algoritmos de manejo que pueden evitar laparotomía tardía o innecesaria. Cada centro los adopta en base a sus recursos y experiencia, y los revisa constantemente para que sus pacientes logren los mejores resultados y la más alta calidad.

Dado que la laparoscopia no es sensible a las lesiones del intestino delgado, el asa intestinal debe inspeccionarse minuciosamente $y$, si se sospecha que hay lesiones involucradas, se convertirá inmediatamente en laparotomía. Cuando se puede observar claramente la lesión y la posición es buena, se puede realizar la reparación laparoscópica, si esto no 
es posible, se puede realizar la reparación auxiliar mediante una incisión abdominal limitada y es suficiente para resolver la reparación laparoscópica. Es mejor utilizar la TC para evaluar la lesión penetrante del abdomen y el costado posterior.

La evaluación y el tratamiento de las heridas abdominales penetrantes ha pasado de la laparotomía obligatoria a un método más selectivo. Se espera reducir la incidencia de cirugía laparoscópica negativa, especialmente en pacientes con hemodinámica estable, que es la principal aplicación del examen laparoscópico. Este concepto se convierte en una menor incidencia de cirugía laparoscópica negativa y permite identificar rápidamente la necesidad de laparotomía exploratoria, reduciendo así la aparición de complicaciones postoperatorias, independientemente de que exista una lesión abdominal. La tecnología de laparoscopia existe desde hace casi un siglo y ha recuperado el interés de los cirujanos. Es una técnica que se utiliza para evaluar a los pacientes que pueden tener un traumatismo abdominal. Su aplicación está sujeta a 
más tipos de pacientes son más precisos y limitan las posibilidades de otros diagnósticos procedimientos. Lesionado. 


\section{Referencias}

1. Ministerio de Salud de Ecuador. Guía Clínica Politraumatizado., Minsal, (2017),

2. L. Medina, R. Kaempffer. Consideraciones epidemiológicas sobre los traumatismos en Chile. Rev. Chil. Cir, 59 (2007), pp. 175-184

3. R. Sanchez, T. Lama, E. Carrillo. Trauma abdominal. En Trauma. Sociedad Panamericana de Trauma. $2^{\mathrm{a}}$, Distribuna Editorial, (2009), pp. 307-315

4. A. González, A. García. Trauma abdominal penetrante. En Trauma. 2a, (2009), pp. 317-328 


\section{Descubre tu próxima lectura}

Si quieres formar parte de nuestra comunidad, regístrate en https://www.grupocompas.org/suscribirse y recibirás recomendaciones y capacitación
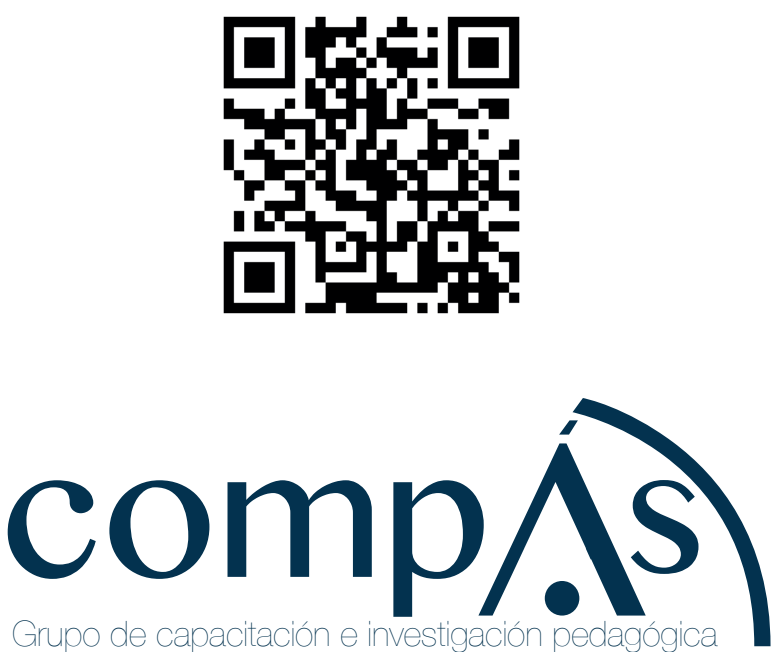

\%

compasacademico@icloud.com 


\section{comp/s}

$5 \bigcirc$ @ @

compasacademico@icloud.com 
